\title{
Chapitre 1 : Pathogénie des Encéphalopathies Spongiformes Transmissibles
}

La compréhension des mécanismes par lesquels la protéine prion pathogène est introduite dans l'organisme, puis la façon dont elle diffuse dans les différents tissus et organes et exerce son pouvoir pathogène est essentielle à bien des égards. C'est notamment le cas pour la mise au point de méthodes de dépistage et de diagnostic, l'évaluation du risque pour la santé publique, la thérapeutique et la connaissance des voies de transmission de la maladie.

Deux types d'approches ont été développées pour étudier ces mécanismes, complémentaires l'une de l'autre. La première est basée sur les modèles animaux. L'article de Schelcher et al analyse les connaissances récentes sur la pathogénie de la tremblante qui découlent des travaux entrepris sur le mouton. Ils portent sur le cheminement de la protéine prion dans l'organisme infecté - dans quels tissus diffuse la protéine prion pathogène, dans quels types de cellules et à quelle vitesse - et comment intervient le génotype de l'individu dans ce processus.

La deuxième approche concerne les modèles cellulaires. L'article de Vilette et Laude présente plusieurs modèles cellulaires mis au point récemment, qui permettent la multiplication du prion ovin. Les différents projets de recherche développés actuellement à partir de ces modèles cellulaires étudient les interactions entre la protéine prion pathogène et différents types de cellules de l'organisme, les gènes activés en cas d'infection, le rôle du polymorphisme de la protéine prion ovine dans la réplication du prion pathogène, et l'identification de molécules ayant une activité antiprion. 